\title{
Analysis of Urinary Pathogen Cultures and Drug Sensitivity in Patients with Urinary Stones for Five Consecutive Years in Xiangya Hospital, China
}

This article was published in the following Dove Press journal: Infection and Drug Resistance

\author{
Yao Bai $\mathbb{D}^{1,2}$ \\ Qingxia Liu $^{3}$ \\ Jie $\mathrm{Gu}^{1,4}$ \\ Xiaobo Zhang' \\ Sheng $\mathrm{Hu}^{\prime}$
}

'Xiangya International Medical Center, Department of Geriatrics Urology, Xiangya Hospital, Central South University, Changsha 410008, People's Republic of China; ${ }^{2}$ Medizinische Klinik and Poliklinik IV, Klinikum Der Universität München, Munich, Germany; ${ }^{3}$ Department of Clinical Laboratory, Xiangya Hospital, Central South University, Changsha 410008, People's Republic of China; ${ }^{4}$ Martini-Klinik Prostate Cancer Center, Department of Urology, University Hospital HamburgEppendorf, Hamburg, Germany
Correspondence: Xiaobo Zhang Xiangya International Medical Center Department of Geriatrics, Xiangya Hospital, Central South University, Changsha 410008, People's Republic of China

Email zhangxb@csu.edu.cn

Sheng Hu

Xiangya International Medical Center,

Department of Geriatrics, Xiangya

Hospital, Central South University,

Changsha 410008, People's Republic of

China

Email I55749722I3@I63.com
Objective: To analyze pathogen distribution and drug sensitivity in patients with urinary calculi and thereby gain insight into the most appropriate antibacterial drugs for perioperative therapy.

Materials and Methods: From January 2014 to December 2018, the results of mid-stream urine pathogen culture and drug sensitivity tests were evaluated retrospectively for 353 patients with urinary calculi. SPSS software version 23.0 was used to analyze the data.

Results: A total of 353 strains of pathogens were isolated from urine culture. Among these, $278(79 \%)$ strains belonged to the top 10 most frequently isolated pathogens, comprising 209 (75.2\%) Gram-negative bacilli and 69 (24.8\%) Gram-positive cocci. Escherichia coli was the most frequently isolated pathogen overall and the most frequently isolated Gram-negative bacillus, and Enterococcus faecalis was the most frequently isolated Gram-positive coccus. Drug sensitivity levels were effectively unchanged for less commonly used drugs, whereas drug resistance rates remained high for commonly used drugs such as ampicillin trihydrate, ampicillin/sulbactam, cefazolin, ceftriaxone, aztreonam, levofloxacin and ciprofloxacin.

Conclusion: E. coli and E. faecalis remain the most common Gram-negative bacillus and Gram-positive coccus uropathogens, respectively, in patients with urinary calculi. Midstream urine pathogen culture and drug sensitivity tests should be used to select appropriate antibacterial drugs before treatment, particularly for perioperative patients with urinary calculi.

Keywords: urine pathogen culture, drug sensitivity, urinary calculi, Escherichia coli, Enterococcus faecalis

\section{Introduction}

Urinary stones and urinary tract infections are common diseases of the urinary system, which can exist alone or simultaneously and can affect each other's disease course. In recent years, studies have shown that pathogens can promote stone formation and urinary tract stones can be secondary to urinary tract infection. ${ }^{1-3}$ Patients with urinary tract infections are more likely to suffer from complicated kidney stones and urinary tract obstruction. Urinary tract obstruction dilatation and effusion are beneficial for the reproduction of pathogens and may thereby lead to urinary tract infection. ${ }^{1}$ Urinary calculi are a common disease in urology, and septic shock is a common cause of death following urinary stone surgery in patients. ${ }^{4-6}$

Effective control of pathogen infections plays an important role in the prevention of perioperative infection and stone recurrence in patients with urinary calculi. 
Determining the distribution of pathogens in urine and the drug sensitivity of these pathogens is crucially important for the treatment of urinary pathogen infection in patients with urinary calculi. Although some scholars ${ }^{7,8}$ previously studied the distribution of pathogens during urinary tract infections and Escherichia coli was reported as the most common pathogen, no similar studies have been published in recent years. The distribution of pathogens during urine infection and the drug sensitivity in patients with urinary stones may be affected by the clinical use of antibacterial drugs over time.

Since antimicrobial drugs were introduced into clinical practice, their improper use and the associated rise in antimicrobial resistance have become significant challenges to human health. ${ }^{9}$ The overuse of antibiotics has been a widespread problem in China and, in recent years, China has introduced many policies and methods to eliminate the abuse of antibiotics. The Ministry of Health of China has attached considerable importance to the appropriate use of antibiotics and to the containment of antimicrobial resistance by issuing a series of policies, specifications and guidelines since the launch of the special corrective action on clinical antimicrobial use in 2011. ${ }^{9}$ It is, therefore, necessary to investigate whether there has been any change in the distribution of pathogens or the tolerance to drugs in recent years.

In this study, we analyzed data from the last 5 years on the distribution of pathogens and drug sensitivity in patients with urinary calculi. The aim of this study was to gain an understanding of the most common pathogens detected in such patients during the perioperative period, so that clinicians can select the most appropriate drugs for treatment, thereby reducing the likelihood of death due to septic shock.

\section{Materials and Methods}

\section{Basic Information}

From January 2014 to December 2018, 353 patients with urinary calculi in our hospital were reported to have a pathogen infection based on urine testing. Of these patients, 187 were male and 217 were female. Their ages ranged from 22 to 84 years, with an average age of 53.82 \pm 12.14 years. There were 192 cases of ureteral stones, 78 cases on the left side, 96 cases on the right side and 18 cases on both sides. There were 159 cases of kidney stones, 73 cases on the left side, 80 cases on the right side and 6 cases on both sides. Ampicillin trihydrate, levofloxacin and ceftazidime are most frequently administered to the enrolled patients. There were also two cases of bladder stones and no cases of urethral stones. Clean catch sampling was used to collect clean mid-stream urine into a sterile tube, which was then immediately sent to the microbiology laboratory for quantitative culture. Urine was usually sampled in the morning before antibacterial treatment.

\section{Urine Culture and Drug Sensitivity Test}

For urine specimens with suspected bacterial infection, $1 \mu \mathrm{L}$ of the sample was inoculated onto a blood agar plate and cultured at $35-37^{\circ} \mathrm{C}$ for $18-24 \mathrm{~h}$. For urine specimens with suspected fungal infection, $1 \mu \mathrm{L}$ of the sample was inoculated onto Agar medium and cultured at $28^{\circ} \mathrm{C}$ for 7 days. The results were analyzed by mass spectrometry according to the characteristics of the colonies, including stainability and morphology, under a microscope.

Then, the microbroth dilution method was carried out for drug sensitivity testing. Standard operating procedures were followed and the MIC reference range for specific pathogenic bacterial colonies was based on the Performance Standards for Antimicrobial Susceptibility. ${ }^{10-14}$ Quantitative analysis was carried out on the drug sensitivity of the pathogens. The same pathogens in the same patient were only included for the first drug sensitivity results.

The main reagents used in the drug sensitivity test were the drug-sensitive reaction card (biomerieux Company, Craponne, France) and the Figure 3 yeast-like fungal drugsensitive reaction strip (biomerieux Company). The equipment used for the drug sensitivity test included a common incubator (Hangzhou Lefeng Technology) and a bacterial turbidimeter (biomerieux Company).

\section{Statistical Analysis}

Data are shown as the mean \pm standard deviation (SD). SPSS23.0 software was used to analyze the data. The Chisquared test was used to compare differences in categorical data between two groups. $\mathrm{P}<0.05$ was considered to indicate a statistically significant difference.

\section{Results}

\section{Composition of Pathogenic Organisms Isolated from the Urine Culture of Patients with Urinary Stones}

From 2014 to 2018 in the urology department of our hospital, a total of 353 strains of pathogenic organisms were isolated from the urine culture of patients with urinary stones. The 
A Distribution of pathogens

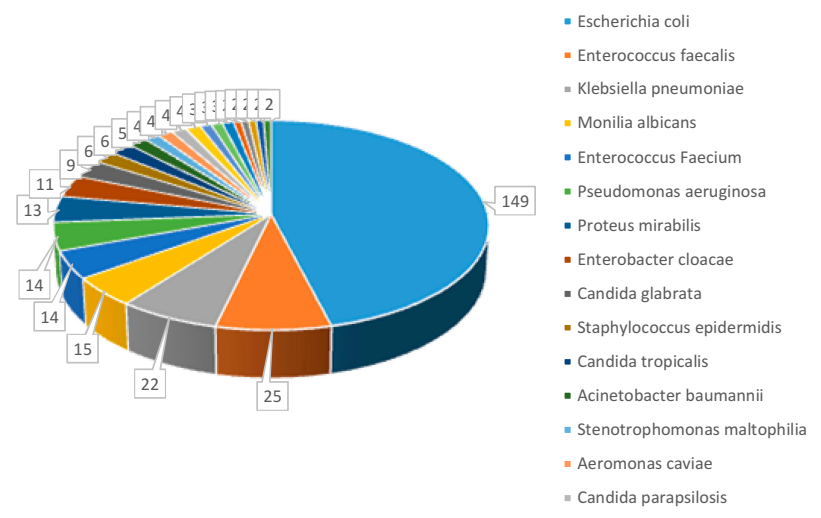

B Distribution of pathogens

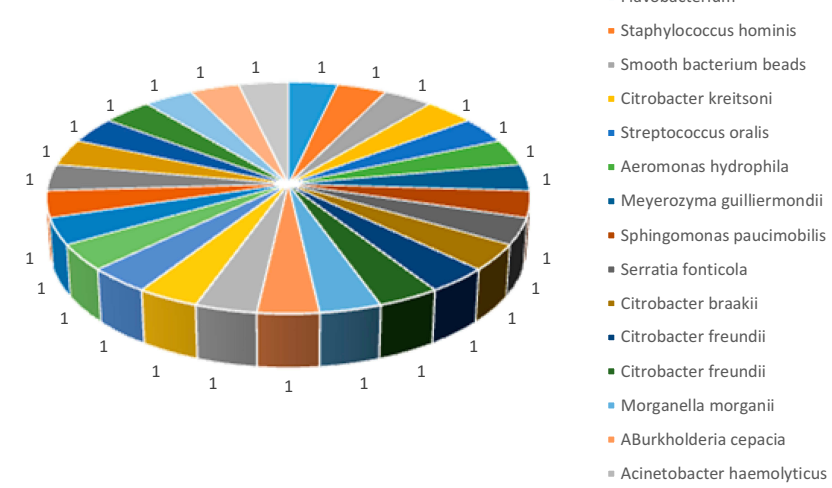

Figure I Distribution of pathogens over the 5-year period from 2014 to 2018 (A, B).

types and proportions of pathogens detected are shown in Figures 1 and 2. Among the 353 isolated strains, 278 (79\%) strains belonged to the top 10 most frequently isolated pathogens, comprising 209 (75.2\%) Gram-negative bacilli and 69 (24.8\%) Gram-positive cocci, as shown in Table 1. The detection rates over the 5-year period of Gram-negative bacilli and Gram-positive cocci are shown in Figure 3, and no obvious differences were observed between the years. The detection rate of Enterococcus faecalis reached a peak in 2016 and then returned to previous levels. The overall detection rate of Escherichia coli showed a downward trend, reaching its lowest level in 2018.

\section{Phenotypic Analysis of Pathogens}

Among the Enterobacteriaceae isolates, extended-spectrum beta-lactamase (ESBL) producers were identified in 22 $(15 \%)$ and $15(10.2 \%)$ of the E. coli and Klebsiella pneumoniae isolates, respectively. The sensitivities of both Gram-negative bacilli and Gram-positive cocci to commonly used antibacterial drugs are shown in Tables 2 and
3. Ampicillin trihydrate has been linked with the highest incidence of drug resistance among all of the antibiotics over the years. Overall, the drug resistance rates in the Gramnegative bacillus group were significantly higher than in the Gram-positive coccus group. Among them, the drug resistance rates to ampicillin trihydrate, ampicillin/sulbactam, cefazolin, ceftriaxone, aztreonam, levofloxacin, ciprofloxacin and bactrim were higher than $50 \%$ for Gram-negative bacilli. For Gram-positive cocci, the drug resistance rates to ampicillin/sulbactam, tetracycline, erythrocin and clindamycin were also higher than $50 \%$. The sensitivity to most antibiotics has not changed significantly over the last 5 years, with the exception of ampicillin trihydrate, cefepime and gentamicin. The sensitivity to ampicillin trihydrate showed a significant difference between 2014 and 2016, $\chi 2=4.230^{\mathrm{a}} \mathrm{p}=0.048$. The sensitivity to cefepime showed a significant difference between 2014 and 2016, $\chi 2=11.671^{\mathrm{a}} \mathrm{p}=0.002$, and between 2014 and 2017, $\chi 2=11.087^{\mathrm{a}} \mathrm{p}=0.002$. The sensitivity to gentamicin showed a significant difference between 2014 and 2016, $\chi 2=9.011^{\mathrm{a}}$
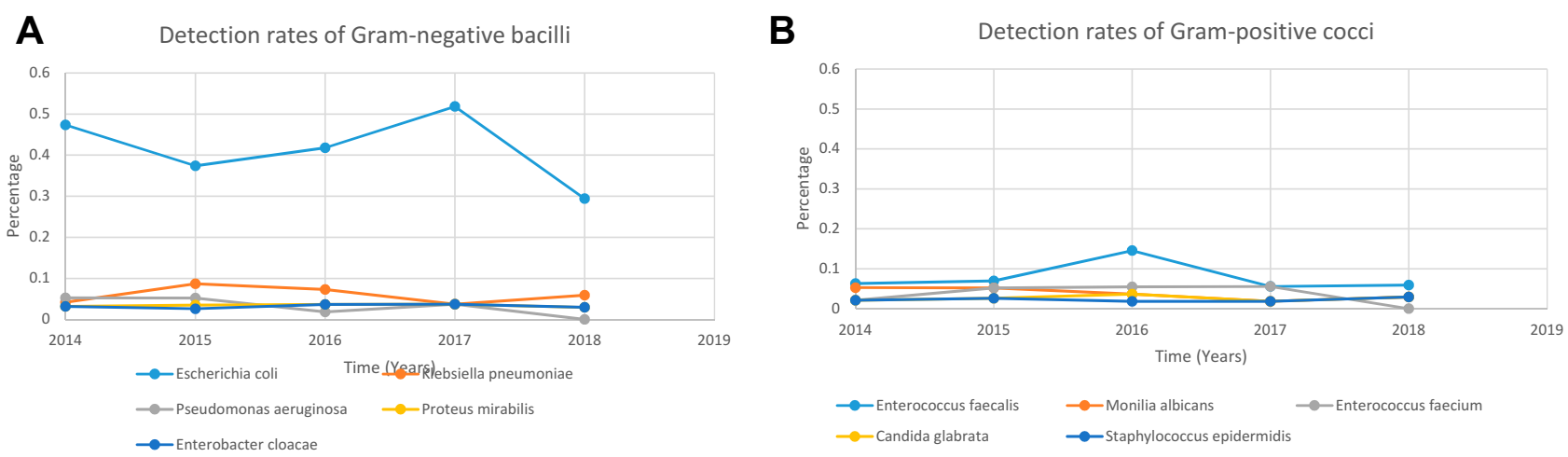

Figure 2 Detection rates of Gram-negative bacilli $(\mathbf{A})$ and Gram-positive cocci $(\mathbf{B})$ over the 5-year period. 
Table I Top 10 Most Frequently Detected Pathogens in Urine Cultures from Patients with Urinary Calculi

\begin{tabular}{|c|c|c|c|}
\hline Pathogens & $\begin{array}{l}\text { Pathogens } \\
\text { Number }\end{array}$ & $\begin{array}{l}\text { Total } \\
\text { Proportion }\end{array}$ & $\begin{array}{l}\text { Proportion of } \\
\text { GN/GP Bacillus }\end{array}$ \\
\hline Escherichia coli & 149 & $53.6 \%$ & $71.3 \%^{\mathrm{a}}$ \\
\hline $\begin{array}{l}\text { Enterococcus } \\
\text { faecalis }\end{array}$ & 25 & $9.0 \%$ & $36.2 \%^{b}$ \\
\hline $\begin{array}{l}\text { Klebsiella } \\
\text { pneumoniae }\end{array}$ & 22 & $7.9 \%$ & $10.5 \%^{\mathrm{a}}$ \\
\hline $\begin{array}{l}\text { Monilia } \\
\text { albicans }\end{array}$ & 15 & $5.4 \%$ & $21.7 \%^{b}$ \\
\hline $\begin{array}{l}\text { Enterococcus } \\
\text { faecium }\end{array}$ & 14 & $5.0 \%$ & $20.3 \%^{\mathrm{b}}$ \\
\hline $\begin{array}{l}\text { Pseudomonas } \\
\text { aeruginosa }\end{array}$ & 14 & $5.0 \%$ & $6.7 \%^{\mathrm{a}}$ \\
\hline $\begin{array}{l}\text { Proteus } \\
\text { mirabilis }\end{array}$ & 13 & $4.7 \%$ & $6.2 \%^{\mathrm{a}}$ \\
\hline $\begin{array}{l}\text { Enterobacter } \\
\text { cloacae }\end{array}$ & 11 & $4.0 \%$ & $5.3 \%^{\mathrm{a}}$ \\
\hline $\begin{array}{l}\text { Candida } \\
\text { glabrata }\end{array}$ & 9 & $3.2 \%$ & $13.1 \%^{b}$ \\
\hline $\begin{array}{l}\text { Staphylococcus } \\
\text { epidermidis }\end{array}$ & 6 & $2.2 \%$ & $8.7 \%^{\mathrm{b}}$ \\
\hline
\end{tabular}

Abbreviations: a GN, Gram-negative; ${ }^{\text {b}} \mathrm{GP}$, Gram-positive.

$\mathrm{p}=0.002$, between 2015 and 2016, $\chi 2=12.718^{\mathrm{a}} \mathrm{p}<0.001$, and between 2016 and 2017, $\chi^{2}=10.218^{\mathrm{a}} \mathrm{p}=0.001$.

\section{Discussion}

In our study, we found that $E$. coli was the most commonly isolated Gram-negative bacillus in urinary system infections, followed by the Gram-positive coccus E. faecalis. The top 10 most commonly isolated pathogens in urinary infections were E. coli, E. faecalis, K. pneumoniae, Monilia albicans, Enterococcus faecium, Pseudomonas aeruginosa, Proteus mirabilis, Enterobacter cloacae, Candida glabrata and Staphylococcus epidermidis, which was consistent with a previous study. ${ }^{7}$ It is worth noting that although the most frequently detected pathogens remain consistent, their rank may alter over time. We found that $E$. coli still ranked highest but the detection rate of $E$. faecalis gradually increased to second place.

The levels of antibacterial resistance are high in China due to the overuse of antibiotics. ${ }^{15,16}$ The results of our study showed a wide range of pathogens responsible for urine infections in patients with urinary tract stones, of which 78.5\% were Gram-negative bacilli. In this study, the drug sensitivity of Gram-negative bacilli to imipenem and amikacin was higher than $95 \%$, and sensitivity to cefepime, nitrofurantoin and cefoperazone/sulbactam was higher than $85 \%$. The rate of drug sensitivity to commonly used antibacterials such as ampicillin, ampicillin/sulbactam, cefazolin, ciprofloxacin and levofloxacin was less than 50\%. Taken together, the drug resistance rates among urinary infection strains were significant in patients with urinary stones. Quinolones and fluoroquinolones are reported to be ineffective at countering uropathogenic E. coli infections, ${ }^{17}$ and in our study, E. coli also showed low drug sensitivity to quinolones. It is suggested that quinolone antibacterial drugs should not be used as first-line empiric drugs in the treatment of urinary infections before the results of drug sensitivity tests are reported.

A series of studies have shown that enterococcal infections usually occur in patients who have received multiple antibiotics, had nosocomial infections or have shown

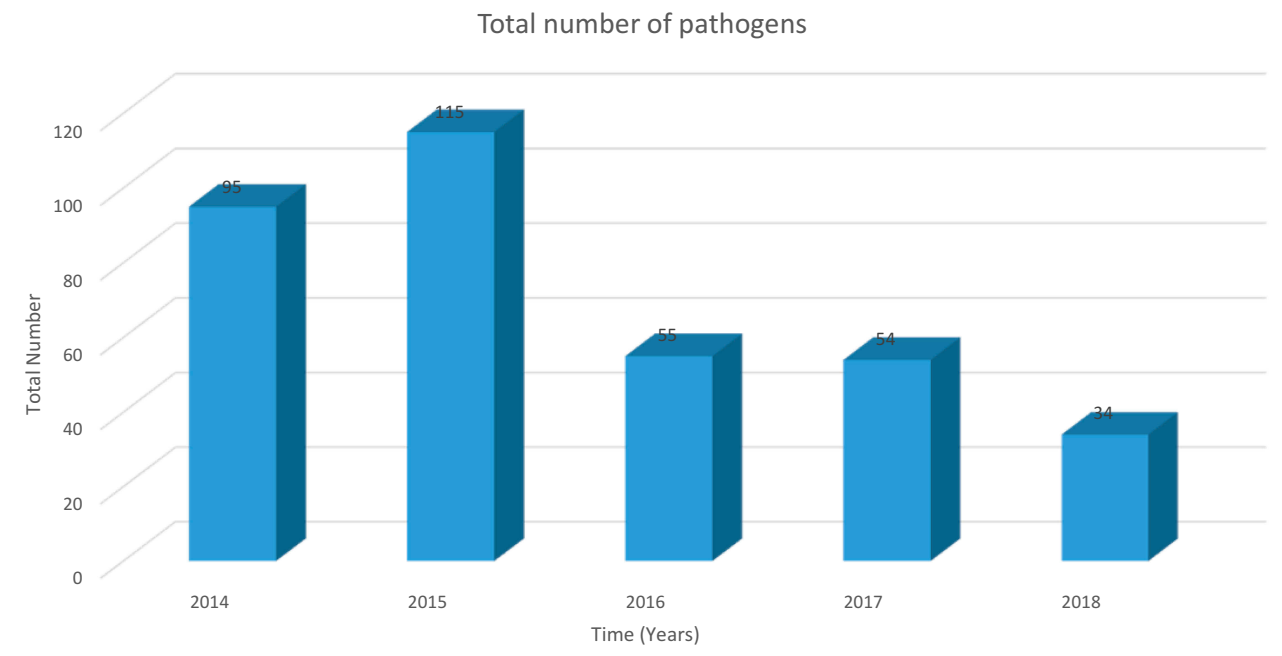

Figure 3 The total number of pathogens detected over the 5-year period. 
Table 2 Sensitivity of the Major Gram-Negative E. Coli Isolates to Commonly Used Antibacterial Drugs (\%)

\begin{tabular}{|c|c|c|c|c|c|c|c|c|c|c|}
\hline \multicolumn{11}{|l|}{ Escherichia coli } \\
\hline \multirow[t]{2}{*}{ Antibacterial Drugs } & \multicolumn{2}{|c|}{2014} & \multicolumn{2}{|c|}{2015} & \multicolumn{2}{|c|}{2016} & \multicolumn{2}{|c|}{2017} & \multicolumn{2}{|c|}{2018} \\
\hline & $\mathbf{S}$ & $\mathbf{I}$ & $\mathbf{s}$ & $\mathbf{I}$ & $\mathbf{S}$ & $\mathbf{I}$ & $\mathbf{S}$ & $\mathbf{I}$ & $\mathbf{S}$ & $\mathbf{I}$ \\
\hline Ampicillin Trihydrate & 16 & 9 & 21 & 3 & 0 & 4 & 18 & 0 & 10 & 0 \\
\hline Ampicillin/Sulbactam & 36 & 13 & 36 & 8 & 17 & 13 & 36 & 7 & 30 & 20 \\
\hline Cefazolin & 42 & 0 & 41 & 0 & 43 & 0 & 29 & 4 & 40 & 10 \\
\hline Ceftriaxone & 44 & 0 & 38 & 0 & 48 & 0 & 29 & 0 & 50 & 0 \\
\hline Cefoperazone/Sulbactam & 93 & 0 & 95 & 0 & 83 & 4 & 93 & 4 & 100 & 0 \\
\hline Ceftazidime & 69 & 2 & 79 & 3 & 74 & 0 & 71 & 0 & 70 & 0 \\
\hline Cefepime & 98 & 0 & 92 & 0 & 70 & 0 & 71 & 0 & 90 & 0 \\
\hline Aztreonam & 58 & 0 & 38 & 31 & 48 & 0 & 50 & 4 & 70 & 0 \\
\hline Imipenem & 91 & 2 & 97 & 0 & 91 & 0 & 96 & 0 & 100 & 0 \\
\hline Gentamicin & 67 & 2 & 59 & 0 & 100 & 0 & 64 & 0 & 70 & 10 \\
\hline Amikacin & 98 & 0 & 95 & 0 & 96 & 0 & 100 & 0 & 100 & 0 \\
\hline Levofloxacin & 40 & 4 & 46 & 0 & 26 & 0 & 29 & 7 & 70 & 0 \\
\hline Ciprofloxacin & 38 & 0 & 41 & 0 & 22 & 4 & 32 & 0 & 60 & 0 \\
\hline Bactrim & 56 & 0 & 56 & 0 & 57 & 0 & 46 & 0 & 80 & 0 \\
\hline Nitrofurantoin & 84 & 4 & 92 & 3 & 78 & 9 & 89 & 7 & 100 & 0 \\
\hline
\end{tabular}

Abbreviations: S, susceptible I, intermediate.

a delayed disease course, and that infecting enterococci are multidrug-resistant. ${ }^{18-20}$ Our results showed that E. faecalis is more sensitive to nitrofurantoin, ciprofloxacin, chloramphenicol and moxifloxacin, indicating that these drugs can be used as first-line drugs for E. faecalis.

The effective control of perioperative urinary calculi infection along with urinary tract infection is critical and the choice of antibacterial drug is particularly important. Cases with urinary tract stones can become infected due to obstruction of the urinary tract, and the pus kidney may appear, potentially leading to loss of renal function. If lithotripsy is received in the kidney pelvis or in the ureter, it can induce bacteremia or sepsis, which can be lifethreatening due to pathogens released from the stones entering the bloodstream via the wound. ${ }^{21,22}$ In addition, Margel et $\mathrm{al}^{23}$ reported that pathogen cultures of the calculi show a few differences between the pre-urine and intraoperative samples. However, pathogen culture from

Table 3 Sensitivity of the Major Gram-Positive E. Faecalis Isolates to Commonly Used Antibacterial Drugs (\%)

\begin{tabular}{|c|c|c|c|c|c|c|c|c|c|c|}
\hline \multirow[t]{3}{*}{ Antibacterial Drugs } & \multicolumn{10}{|c|}{ Enterococcus faecalis } \\
\hline & \multicolumn{2}{|c|}{2014} & \multicolumn{2}{|c|}{2015} & \multicolumn{2}{|c|}{2016} & \multicolumn{2}{|c|}{2017} & \multicolumn{2}{|c|}{2018} \\
\hline & $\mathbf{S}$ & $\mathbf{I}$ & $\mathbf{S}$ & $\mathbf{I}$ & $\mathbf{S}$ & $\mathbf{I}$ & $\mathbf{S}$ & $\mathbf{I}$ & $\mathbf{S}$ & $\mathbf{I}$ \\
\hline Ampicillin Trihydrate & 50 & 0 & 63 & 0 & 75 & 0 & 40 & 0 & 100 & 0 \\
\hline Ampicillin/Sulbactam & 33 & 0 & 75 & 0 & 100 & 0 & 80 & 0 & 100 & 0 \\
\hline Penicillin & 83 & 0 & 88 & 0 & 75 & 0 & 40 & 0 & 80 & 0 \\
\hline Gentamicin & 67 & 2 & 75 & 0 & 75 & 0 & 80 & 0 & 80 & 0 \\
\hline Levofloxacin & 40 & 4 & 100 & 0 & 75 & 0 & 40 & 0 & 80 & 0 \\
\hline Nitrofurantoin & 100 & 0 & 88 & 0 & 100 & 0 & 40 & 20 & 100 & 0 \\
\hline Tetracycline & 50 & 0 & 50 & 0 & 0 & 0 & 20 & 0 & 60 & 20 \\
\hline Erythrocin & 67 & 0 & 38 & 0 & 13 & 25 & 20 & 0 & 60 & 0 \\
\hline Ciprofloxacin & 100 & 0 & 100 & 0 & 50 & 13 & 40 & 0 & 80 & 0 \\
\hline Chloramphenicol & 100 & 0 & 100 & 0 & 100 & 0 & 100 & 0 & 100 & 0 \\
\hline Clindamycin & 83 & 0 & 50 & 0 & 0 & 0 & 20 & 0 & 60 & 0 \\
\hline Moxifloxacin & 100 & 0 & 100 & 0 & 50 & 13 & 40 & 0 & 80 & 0 \\
\hline
\end{tabular}

Abbreviations: S, susceptible; I, intermediate. 
stones is not always effective, and the results of drug sensitivity tests can usually be obtained 2-3 days after surgery. Most infected patients will develop symptoms such as a high fever within 24 hours of surgery. The bacteria may not be sensitive to commonly used antibacterial drugs and the treatment of infection based on empiric medication is not sufficient; therefore, urine culture and drug sensitivity tests are necessary prior to treatment. Pathogen culture from stones is important to guide the rational selection of antibiotics in clinical practice and to effectively control urinary tract infections and postoperative infections in patients with urinary tract stones.

\section{Conclusion}

The rate of urinary infections has decreased in recent years along with greater control of antibiotic use in China. E. coli and E. faecalis are still the most common uropathogens of Gram-negative bacilli and Gram-positive cocci, respectively, that cause urinary infections in patients with urinary calculi. Mid-stream urine pathogen culture and drug sensitivity tests should be used to select appropriate antibacterial drugs before treatment, particularly for perioperative patients with urinary calculi. Our findings may help clinicians to take adequate precautions to reduce the risk of death in these patients.

\section{Ethics Approval}

The study was approved by the Ethics Committee of Xiangya Hospital of Central South University, Changsha, China (No. 2018121161). The ethics committee waived the need for written informed consent provided by participants due to the retrospective nature of this study. Because all patient data were analyzed in anonymity, no additional informed consent was required.

\section{Acknowledgments}

This work was supported by the Fundamental Research Funds for the Central Universities of Central South University (grant no. 2019zzts1037, Changsha, China). YB and JG were supported by a scholarship from the China Scholarship Council Program (CSC). We thank Kate Fox, DPhil, from Liwen Bianji, Edanz Group China, for editing the English text of a draft of this manuscript.

\section{Disclosure}

The authors report no conflicts of interest in this work.

\section{References}

1. Miano R, Germani S, Vespasiani G. Stones and urinary tract infections. Urol Int. 2007;79(1):32-36. doi:10.1159/000104439

2. Xu G, He Y, Zhao H, et al. Mini-nephroscope combined with pressure suction: an effective tool in MPCNL for intrarenal stones in patients with urinary tract infections. Urolithiasis. 2016;44 (5):445-450. doi:10.1007/s00240-016-0859-8

3. Shi B, Hong X, Yu J. Management of unilateral staghorn renal stones with concurrent urinary tract infections by retroperitoneal laparoscopic pyelolithotomy with prolonged renal posterior lower segment incision. Exp Ther Med. 2019. doi:10.3892/etm.2019.7545

4. Li K, Liu C, Zhang X, Liu Y, Wang P. Risk factors for septic shock after mini-percutaneous nephrolithotripsy with holmium laser. Urology. 2013;81(6):1173-1176. doi:10.1016/j.urology.2013.01.035

5. Yang X, Zhang D, Li Y, Chong T, Zhang P. Analysis on the risk factors for septic shock following minimally invasive percutaneous nephrolithotomy. Int J Clin Exp Med. 2018;11(5):4925-4930.

6. Wang CT, Hao B. Associated factors of SIRS and residual stones after flexible ureteroscopic holmium laser lithotripsy for calyceal diverticular stones. Chin Gen Pract. 2017;20(17):2079-2084.

7. Ma K, Xu QQ, Huang XB, et al. [Analysis and clinical implication of upper urinary tract stone's bacterial spectrum]. Zhonghua Wai Ke Za Zhi. 2010;48(4):293-295.

8. Dajani AM, Khadra ALIA. Urinary calculi and urinary tract infection. In: Urolithiasis. Boston, MA:Springer; 1989:277-278.

9. Zhou J, Ma X. A survey on antimicrobial stewardship in 116 tertiary hospitals in China. Clin. Microbiol. Infect. 2019;25(6):759.e9-759. e14. doi:10.1016/j.cmi.2018.09.005

10. Clinical and Laboratory Standards Institute. Performance Standards for Antimicrobial Susceptibility Testing; Twenty-First Informational Supplement Clinical and Laboratory Standards Institute; 2011.

11. CLSI. Performance Standards for Antimicrobial Susceptibility Testing; Twenty-Second Informational Supplement Clinical and Laboratory Standards Institute; 2015.

12. CLSI. Performance standards for antimicrobial susceptibility testing: 26th informational supplement, document M100-S26. Clin Lab Stand Inst. 2016.

13. CLSI. Performance Standards for Antimicrobial Susceptibility Testing. 27th. CLSI supplement M100. Wayne, PA:: Clinical and Laboratory Standards Institute; 2017

14. CLSI. Performance standards for antimicrobial susceptibility testing performance standards for antimicrobial susceptibility testing suggested citation. In: CLSI Doc. M02-A11. 2018.

15. Zeng S, Xu Z, Wang X, et al. Time series analysis of antibacterial usage and bacterial resistance in China: observations from a tertiary hospital from 2014 to 2018. Infect Drug Resist. 2019;12:2683-2691. doi:10.2147/IDR.S220183

16. Zhang X, Lu Q, Liu T, Li Z, Cai W. Bacterial resistance trends among intraoperative bone culture of chronic osteomyelitis in an affiliated hospital of South China for twelve years. BMC Infect Dis. 2019;19 (1). doi:10.1186/s12879-019-4460-y

17. Singh BR. Quinolones and fluoroquinolones are useless to counter uropathogenic Escherichia coli infections [letter]. Infect Drug Resist. 2019;12:2161-2162. doi:10.2147/IDR.S220394

18. Chen L, Bu Q, Xu H, et al. The effect of berberine hydrochloride on Enterococcus faecalis biofilm formation and dispersion in vitro. Microbiol Res. 2016;186-187:44-51. doi:10.1016/j. micres.2016.03.003

19. Yan Q, Karau MJ, Patel R. In vitro activity of oritavancin against planktonic and biofilm states of vancomycin-susceptible and vancomycin-resistant enterococci. Diagn Microbiol Infect Dis. 2018;91(4):348-350. doi:10.1016/j.diagmicrobio.2018.03.008

20. Liu J, Huang X, Liu Y, Xu H, Gong R, Li C. Clinical analysis for patients with continuous ambulatory peritoneal dialysis associated peritonitis. J Cent South Uni. 2016;41(12):1328-1333. 
21. Kreydin EI, Eisner BH. Risk factors for sepsis after percutaneous renal stone surgery. Nat Rev Urol. 2013;10(10):598-605. doi:10.1038/nrurol.2013.183

22. Marien T, Miller NL. Treatment of the infected stone. Urol Clin North Am. 2015;42(4):459-472. doi:10.1016/j.ucl.2015.05.009
23. Margel D, Ehrlich Y, Brown N, Lask D, Livne PM, Lifshitz DA. Clinical implication of routine stone culture in percutaneous nephrolithotomy - A prospective study. Urology. 2006;67(1):26-29. doi:10.1016/j.urology.2005.08.008

\section{Publish your work in this journal}

Infection and Drug Resistance is an international, peer-reviewed openaccess journal that focuses on the optimal treatment of infection (bacterial, fungal and viral) and the development and institution of preventive strategies to minimize the development and spread of resistance. The journal is specifically concerned with the epidemiology of

Submit your manuscript here: https://www.dovepress.com/infection-and-drug-resistance-journa: antibiotic resistance and the mechanisms of resistance development and diffusion in both hospitals and the community. The manuscript management system is completely online and includes a very quick and fair peerreview system, which is all easy to use. Visit http://www.dovepress.com/ testimonials.php to read real quotes from published authors. 